\title{
PROPIEDADES PSICOMÉTRICAS DE LA ESCALA DE ALTERACIÓN DEL COMPORTAMIENTO EN LA ESCUELA (ACE)
} \author{
(SABS) \\ Lidia Sotelo L. ${ }^{1}$, Noemi Sotelo L., Sergio Domínguez L. \\ Universidad Nacional Mayor de San Marcos, Perú \\ (Recibido el 14/02/2011 - Aceptado el 09/06/2011)
}

Psychometric properties of the scale of alteration of the behavior in the school

\begin{abstract}
RESUMEN
El objetivo de este estudio fue analizar las propiedades psicométricas de la Escala de Alteración del Comportamiento en la Escuela en una muestra de niños peruanos de 1 . $^{\text {er }}$ a $6 .^{\text {to }}$ grado de educación primaria, y obtener evidencias que apoyen su utilización como instrumento de screening para trastornos conductuales (TC). Se obtuvo una Confiabilidad aceptable a través del método de consistencia interna (alpha de Cronbach de 0,937), así como evidencia de estructura interna del test, validez factorial (dos factores que explican el $61,986 \%$ de varianza) y de validez criterial. Estos resultados nos dicen que la Escala de Alteración del Comportamiento en la Escuela (ACE) es un instrumento válido y confiable para ser usado como método de valoración de alteraciones conductuales en alumnos del 1. er $^{\text {er }}$ a $6 .{ }^{\text {to }}$ de educación primaria.
\end{abstract}

Palabras clave: Alteración del comportamiento, niños, evaluación, confiabilidad, validez.

\begin{abstract}
The objective of this study was to analyze the psychometric properties of the Scale of Alteration of the Behavior in the School (SABS) in a sample of Peruvian children of $1^{\circ}$ to $6^{\circ}$ degree of elemental education, and to obtain evidences that support their use like instrument of screening for behavioural alterations (BA). One obtained an acceptable Reliability through method of internal consistency (alpha de Cronbach $=0.937$ ), as well as evidence of internal structure of the test, factorial validity (two factors that explain $61.986 \%$ of variance) and of criterial validity. To these they say us results that the Scale of Alteration of the Behavior in the School (SABS) is an instrument valid and reliable to be used like method of valuation of behavioural alterations in students of $1 .^{\circ}$ to $6 .^{\circ}$ of primary education.
\end{abstract}

Keywords: Alteration of the behavior, children, evaluation, reliability, validity. 


\section{INTRODUCCIÓN}

Los trastornos de la conducta en el niño se consideran como un patrón de comportamiento persistente a lo largo del tiempo que afecta los derechos de otros y violenta las normas apropiadas a la edad, lo cual afecta de manera negativa su relación con el medio circundante. Marsh y Graham (2002) mencionan que los trastornos infantiles se han considerado habitualmente como desviaciones que implican una disminución del funcionamiento adaptativo, una desviación estadística, malestar o incapacidad inesperada y/o deterioro biológico, así como también un fracaso adaptativo, el cual se manifiesta en la desviación del niño de la normas apropiadas para su edad, una exageración de tendencias evolutivas normales, una interferencia en el proceso evolutivo normal o un fracaso en conseguir una función o un mecanismo evolutivo específico; no obstante, es necesaria una comprensión cabal tanto de la conducta del niño como del contexto en el cual se desarrolla para poder determinar si es o no una desviación respecto a la norma; algo contrario a las conductas prosociales, las cuales se refieren a las acciones que tratan de ayudar o beneficiar a otra persona o grupo sin esperar recompensas externas (García, 2008).

Para los manuales diagnósticos más usados, el DSM-IV-TR y el CIE-10, existen tres y seis tipos de trastornos conductuales respectivamente. El DSM-V-TR considera como trastornos del comportamiento perturbador al trastorno disocial, el trastorno negativista desafiante, y trastorno por comportamiento perturbador no especificado; por otro lado, el CIE-10 postula seis categorías, las cuales son: trastorno de conducta limitado al ambiente familiar, trastorno de conducta antisocial, trastorno de conducta socializado, trastorno oposicionista desafiante, otros trastornos de conducta y trastorno de conducta no especificado. Asimismo, existen formatos de entrevista diagnóstica estructurada basados en dichos manuales (Ezpeleta, 2001).

El trastorno de conducta antisocial del CIE-10 corresponde al trastorno disocial del DSM-IV-TR; y el trastorno oposicionista desafiante del CIE-10 al trastorno negativista desafiante del DSM-IV-TR.

Dichos trastornos conductuales se asocian a comportamientos agresivos (violencia, oposición), mentira, robo, fugas (irse sin autorización), y enfocados al contexto escolar es común observar conductas de abuso con los compañeros, incluso con los docentes.

En nuestro contexto, dada la creciente necesidad de contar con un instrumento estandarizado con las características psicométricas adecuada (confiabilidad y validez) para examinar la alteración del comportamiento del alumno, que sea de fácil aplicación para el maestro y que tenga normas conforme a este contexto, se plantea este trabajo, el cual nos brindará las pautas para hacer una valoración más apropiada del examinado. En ese sentido, exploraremos las características psicométricas de la Escala de Alteración del Comportamiento en la Escuela (ACE) de Arias, Ayuso, Gil y González (2009) para evaluar trastornos conductuales en una muestra de alumnos del1 er $^{\text {el }} 6 .{ }^{\text {to }}$ de educación primaria.

La relevancia de este trabajo radica fundamentalmente en su utilidad no sólo para el psicólogo, sino también para los profesionales del área de educación, ya que será una herramienta adecuada para realizar una detección rápida de aquellos alumnos que presenten alteraciones del comportamiento, y en qué nivel de dicha alteración se encuentran. 
Entonces, nos hacemos la siguiente pregunta: ¿Es válida y confiable la Escala de Alteración del Comportamiento en la Escuela (ACE) en una muestra de alumnos de $1 .{ }^{\text {er }}$ a $6 .{ }^{\text {to }}$ de educación primaria?

\section{MÉTODO}

Según Hernández, Fernández y Baptista (2003), es un estudio de diseño no experimental de tipo descriptivo. Según León y Montero (2005), sería un estudio instrumental, destinado a la adaptación y estudio de las propiedades psicométricas de un test.

\section{Sujetos}

De acuerdo a la Tabla 1, la muestra de estudio estuvo conformada por 312 alumnos, 160 varones y 152 mujeres, del $1 .^{\text {er }}$ al $6 .^{\text {to }}$ de educación primaria, pertenecientes a escuelas públicas y privadas, 91 y 221, respectivamente. El muestreo utilizado fue de tipo intencional. A continuación, las tablas de distribución de la muestra.

TABLA 1. Características de la muestra según sexo y grado de estudios

\begin{tabular}{cccc}
\hline \multicolumn{4}{c}{ Colegio de Procedencia } \\
\hline Categoría & Público & Privado & Total \\
\hline Varones & 45 & 115 & 160 \\
\hline Mujeres & 46 & 106 & 152 \\
$1{ }^{\text {er }}$ grado & 21 & 51 & 73 \\
$2{ }^{\text {do }}$ grado & 16 & 26 & 42 \\
$3{ }^{\text {er }}$ grado & 20 & 20 & 40 \\
$4{ }^{\text {to }}$ grado & 20 & 52 & 72 \\
$5 .^{\text {to }}$ grado & 6 & 24 & 30 \\
$6 .{ }^{\text {to }}$ grado & 8 & 47 & 55 \\
\hline
\end{tabular}

\section{Técnicas e instrumento}

Se utilizó la Escala de Alteración del Comportamiento en la Escuela (ACE) de Arias, Ayuso, Gil y González (2009). El instrumento mencionado consta de 16 ítems que describen conductas disruptivas que se producen en el aula, cuya valoración es realizada por el profesor a cargo del estudiante, el cual asignará una puntuación del 0 al 4 cada una de las preguntas según le siguiente parámetro: 0 , no presenta nunca esa conducta; 1 , la conducta se produce esporádicamente; 2 , la conducta se produce algunas veces o con poca intensidad; 3 , la conducta se produce muchas veces o con mucha intensidad; 4, la conducta se produce con mucha frecuencia y con gran intensidad. El tiempo de aplicación es variable, pues 
dependerá del alumno a evaluar. Esta escala tiene dos finalidades prioritarias: despistaje o constatación del grado de desviación conductual del alumno; y en segundo lugar, aportar una base para una evaluación posterior.

\section{Procedimiento}

Se procedió a la aplicación de la Escala de Alteración del Comportamiento en la Escuela (ACE) a los docentes responsables de la muestra de la investigación.

El análisis de los datos se llevó a cabo utilizando el paquete estadístico SPSS 17.0.

\section{RESULTADOS}

Confiabilidad: Se analizó por medio de su consistencia interna usando el método del alpha de Cronbach. Obtuvo un índice de 0,937.

Análisis de la estructura interna del test: En esta sección se examinará el grado de asociación entre los ítems que conforman la prueba y el test (Elosua, 2003).

TABLA 2. Coeficientes de correlación ítem-test

\begin{tabular}{lc}
\hline Ítem & Correlación Ítem-Test \\
\hline Ítem 1 & 0,750 \\
Ítem 2 & 0,499 \\
Ítem 3 & 0,443 \\
Ítem 4 & 0,783 \\
Ítem 5 & 0,795 \\
Ítem 6 & 0,784 \\
Ítem 7 & 0,853 \\
Ítem 8 & 0,791 \\
Ítem 9 & 0,682 \\
Ítem 10 & 0,697 \\
Ítem 11 & 0,491 \\
Ítem 12 & 0,424 \\
Ítem 13 & 0,563 \\
Ítem 14 & 0,672 \\
Ítem 15 & 0,757 \\
Ítem 16 & 0,715 \\
\hline
\end{tabular}


Análisis de la dimensionalidad. Para poder entender la dimensionalidad de la escala creada se realizó un análisis factorial de componentes principales con rotación varimax al cumplir con las características requeridas para dicho análisis.

TABLA 3. Valores de los Índices de Ajuste al Análisis Factorial

\begin{tabular}{lcc}
\hline Prueba & Índice & Sig. \\
\hline Determinante & - & $0,000^{*}$ \\
Kaiser-Meyer-Olkin & 0,914 & - \\
Test de Bartlett $\left(\mathrm{x}^{2}\right)$ & 3543,064 & $0,000^{*}$ \\
${ }^{*} \mathrm{p}<0,001$ & & \\
\hline
\end{tabular}

TABLA 4. Varianza Total Explicada por cada Factor

\begin{tabular}{lccc}
\hline \multirow{2}{*}{ Componente } & \multicolumn{3}{c}{ Valores Eigen Iniciales } \\
\cline { 2 - 4 } & Total & \% de Varianza & $\%$ acumulativo \\
\hline Factor 1 & 8,363 & 52,267 & 52,267 \\
Factor 2 & 1,555 & 9,719 & 61,986 \\
\hline
\end{tabular}

TABLA 5. Resultados de la Rotación Varimax del Análisis Factorial en la Escala de Alteración del Comportamiento en la Escuela (ACE)

\begin{tabular}{lcc}
\hline & \multicolumn{2}{c}{ Componente } \\
\hline Ítems & Factor 1 & Factor 2 \\
Ítem 1 & 0,786 & \\
Ítem 2 & & 0,726 \\
Ítem 3 & & 0,783 \\
Ítem 4 & 0,820 & \\
Ítem 5 & 0,835 & \\
Ítem 6 & 0,825 & \\
Ítem 7 & 0,885 & \\
Ítem 8 & 0,834 & \\
Ítem 9 & 0,731 & \\
Ítem 10 & 0,740 & \\
Ítem 11 & 0,540 & \\
Ítem 12 & 0,470 & \\
Ítem 13 & 0,614 & \\
Ítem 14 & 0,715 \\
Ítem 15 & 0,794 \\
Ítem 16 & 0,754 \\
\hline
\end{tabular}




\section{Validez criterial}

Como parte de la labor de investigación se pidió a los profesores que mencionaran cuáles de los alumnos presentaban problemas de comportamiento (conductas desadaptativas en el aula), y en base a eso se calificación en dos grupos: unos con trastornos de conducta (con TC) y otro sin trastornos de conducta ( $\sin$ TC) presentados a continuación.

TABLA 6. Clasificación de los alumnos según la valoración de los docentes

\begin{tabular}{lcc}
\hline Categoría & Frecuencia & Porcentaje \\
\hline Sin TC & 262 & 84,0 \\
con TC & 50 & 16,0 \\
Total & 312 & 100,0 \\
\hline
\end{tabular}

Como se podrá apreciar, un $84 \%$ de los alumnos evaluados no fueron calificados con problemas de comportamiento, mientras que un $16 \%$ sí. Entonces, se espera que haya diferencias estadísticamente significativas entre las puntuaciones de los alumnos con TC y $\sin T C$, para lo cual se usó la prueba $t$ de Student para muestras independientes.

TABLA 7. Diferencias entre alumnos $\sin T C$ y con $T C$

\begin{tabular}{lccccc}
\hline Categoría & Frecuencia & Media & Desv. Estándar & $\boldsymbol{t}(\mathbf{3 1 0})$ & Sig. \\
\hline Sin TC & 262 & 6,69 & 8,557 & $-13,178$ & $0,000^{*}$ \\
con TC & 50 & 25,26 & 11,730 & & \\
$* \mathrm{p}<0,001$ & & & & & \\
\hline
\end{tabular}

La aplicación de la prueba t para muestras independientes indica que los alumnos con TC presentan un puntaje promedio más elevado que los alumnos $\sin T C(t(310)=-13,178$; $\mathrm{p}<0,01$, bilateral).

\section{Construcción de baremos}

Para determinar si es pertinente la elaboración de baremos separados entre varones y mujeres, se usó la prueba $t$ de Student para muestras independientes.

TABLA 8. Diferencias según sexo

\begin{tabular}{lccccc}
\hline Categoría & Frecuencia & Media & Desv. Estándar & $\boldsymbol{t}(\mathbf{3 1 0})$ & Sig. \\
\hline Varones & 160 & 12,43 & 12,353 & 4,530 & $0,000 *$ \\
Mujeres & 152 & 6,76 & 9,481 & & \\
$* \mathrm{p}<0,001$ & & & & & \\
\hline
\end{tabular}


La aplicación de la prueba t para muestras independientes indica que los varones presentan un puntaje promedio más elevado que las mujeres $(t(310)=4,530 ; \mathrm{p}<0,01$, bilateral). Esto quiere decir que se hallaron diferencias significativas entre sexos por lo cual se construyeron normas diferenciadas, tomando en consideración las cuatro categorías de la versión original del test (Arias, Ayuso, Gil y González, 2009): Desviación Conductual No Constatada, Desviación Conductual Ligera, Desviación Conductual Moderada y Desviación Conductual Severa.

TABLA 9. Estadísticos descriptivos de la escala

\begin{tabular}{lcccc}
\hline Sexo & Media & Desv. Estándar & Mínimo & Máximo \\
\hline Varones & 12,43 & 12,353 & 0 & 51 \\
Mujeres & 6,76 & 9,481 & 0 & 46 \\
\hline
\end{tabular}

TABLA 10. Distribución de la muestra clasificada con TC

\begin{tabular}{lccccc}
\hline & \multicolumn{5}{c}{ Con TC } \\
\hline Sexo & $\mathrm{N}$ & \% Total & \%Ligera & \%Moderada & \% Severa \\
\hline Varones & 41 & 26 & 13 & 9 & 4 \\
Mujeres & 9 & 6 & 3 & 2 & 1 \\
\hline
\end{tabular}

En la versión original de la prueba, se usó el criterio epidemiológico, es decir, considerando el porcentaje de alumnos que se encuentran tanto en la categoría de $\operatorname{con} T C$ y $\sin T C$. Dentro del grupo de alumnos clasificados como personas con TC, la sexta parte de los estudiantes que tengas las puntuaciones más elevadas, se clasificarán como con problemas severos; las dos sextas partes, moderado; y el $50 \%$ restantes, con problemas ligeros de comportamiento (Arias, Ayuso, Gil y González, 2009).

TABLA 11. Baremos para Educación Primaria

\begin{tabular}{lll}
\hline Sexo & \multicolumn{1}{c}{ Categoría } & \multicolumn{1}{c}{ Rango } \\
\hline \multirow{4}{*}{ Varones } & Desviación Conductual No Constatada & Menor a 34 \\
& Desviación Conductual Ligera & Entre 34 y 38 \\
& Desviación Conductual Moderada & Entre 39 y 48 \\
& Desviación Conductual Severa & Igual o mayor a 49 \\
\hline \multirow{2}{*}{ Mujeres } & Desviación Conductual No Constatada & Menos de 43 \\
& Desviación Conductual & Igual o mayor a 43 \\
\hline
\end{tabular}




\section{DISCUSIÓN}

En cuanto al coeficiente de confiabilidad alcanzado de 0,937 , aunque ligeramente menor al coeficiente hallado en la versión original $(0,953)$, de acuerdo a la clasificación hecha por Campo-Arias y Oviedo (2008) es bastante aceptable. Debemos tener en consideración, tal como lo apuntan Prieto y Delgado (2010), que los estadísticos de fiabilidad varían entre poblaciones y están afectados por otras condiciones como la longitud de la prueba y la variabilidad de las muestras de personas. En consecuencia, se ha de evitar el error de considerar que la estimación de la fiabilidad procedente de un único estudio refleja la verdadera y única fiabilidad de la prueba.

En la obtención de evidencias de validez por medio de su estructura interna (Tabla 2), se hallaron correlación que oscilaban entre 0,424 (ítem 12) y 0,791 (ítem 8), lo cual da cuenta de una buena estructura interna, ya que las relaciones entre los ítems y los componentes del test conforman el constructo que se quiere medir y sobre el que se basarán las interpretaciones (Elosua, 2003).

Respecto al análisis de la dimensionalidad del test, se usó el análisis factorial debido a que se cumplieron con las condiciones necesarias (Tabla 3), es decir, una matriz de correlaciones significativa (Determinante significativo al $\mathrm{p}<0,01$ ); índice de KaiserMeyer-Olkin de 0,914, superior a 0,80 (Ferrando y Anguiano, 2010) y test de esfericidad de Bartlett significativo $(\mathrm{p}<0,01)$. Se pudieron extraer dos factores con valor eigen aceptable (eigen value $>1$ ), los cuales explican juntos el $61,986 \%$ de la varianza de la prueba (Tabla 4). El análisis de la dimensionalidad nos dice que el primer factor explica el $52,267 \%$ de la varianza de la prueba, superando el mínimo requerido que es $20 \%$. Esto difiere con el proceso de validación de la escala original, en la cual predominaron tres factores, explicando el $46,67 \%, 8,081 \%$ y $5,287 \%$ respectivamente, haciendo un total de $60,038 \%$ de varianza explicada. Asimismo, 14 de los 16 ítems cargan en el primer factor (Tabla 5), y dos ítems (ítem 2 e ítem 3) en un segundo factor. Para rescatar este último punto diremos que el primer factor está enfocado en las relaciones entre el alumnado; y el segundo factor, por las relaciones entre el profesor y el alumno (ítem 2: Se enfrenta al profesor; ítem 3: Se burla o parece tomarle el pelo al profesor). Entonces, de acuerdo con Elosua (2003), a través de un número mínimo de factores y mayor varianza explicada se trata de proponer un modelo independiente, por lo cual podemos decir que hay evidencias de validez factorial (dimensionalidad).

En el proceso de obtención de evidencias por validez criterial, los resultados y el nivel de significación $(\mathrm{p}<0,01)$ obtenidos con la prueba t de Student (Tabla 7), quiere decir que hay diferencias significativas en el puntaje global de la Escala de Alteración del Comportamiento en la Escuela (ACE) entre alumnos calificados por sus profesores responsables como con problemas de conducta y sin problemas de conducta, es decir, la escala sirve para discriminar entre estos dos grupos bien diferenciados.

En cuanto a los baremos, al hallarse diferencias significativas de acuerdo al sexo (Tabla 8), se procedió a la elaboración de baremos independientes según dicha variable. La distribución porcentual de los alumnos clasificados con TC (Tabla 10), también se han hallado diferencias, ya que los puntos de corte en la validación española son más bajos 
que los de la muestra peruana, por ejemplo, un varón que obtiene 28 como puntuación global, puede estar en la categoría "Desviación conductual ligera" conforme a los baremos españoles, pero en "Desviación conductual no constatada" con las normas obtenidas en el presente estudio (Tabla 11). Esto nos lleva a decir que son importantes las consideraciones contextuales en las cuales se dan ciertos comportamientos. Además, evita la aparición de "falsos positivos" al usar normas que no corresponden a nuestra realidad. Por otro lado, la reducida muestra de alumnas clasificadas con TC imposibilitó que se amplíen las categorías como sus pares masculinos, quedando solo dos (Desviación Conductual No Constatada y Desviación Conductual).

Entonces, de acuerdo a las evidencias la Escala de Alteración del Comportamiento en la Escuela (ACE) es un instrumento válido y confiable para ser usado como método de valoración de alteraciones conductuales en alumnos del $1 .{ }^{\text {er }}$ al $6 .{ }^{\text {to }}$ de educación primaria.

Es recomendable seguir ampliando la muestra de estudio en otras zonas de nuestro país, ya que como sabemos, cada contexto tiene sus propios estándares sociales, costumbres y creencias.

\section{CONCLUSIONES}

1. La Escala de Alteración del Comportamiento en la Escuela (ACE) presentó un nivel de confiabilidad bastante aceptable $(0,937)$.

2. Se obtuvo evidencias de validez por medio de su estructura interna, es decir, por la relación de cada uno de los ítems de la Escala de Alteración del Comportamiento en la Escuela (ACE) con la escala total.

3. La Escala de Alteración del Comportamiento en la Escuela (ACE) presentó evidencias de validez factorial, resultando dos factores que explican juntos el 61,986\% de la varianza de la prueba. El primer factor hallado está enfocado en las relaciones entre el alumnado; y el segundo factor, por las relaciones entre el profesor y el alumno.

4. En la Escala de Alteración del Comportamiento en la Escuela (ACE) se hallaron evidencias de validez criterial, dado que se hallaron hay diferencias significativas $(p<0,01)$ entre alumnos calificados por sus profesores responsables como con problemas de conducta y sin problemas de conducta

5. Se hallaron diferencias significativas de acuerdo al sexo en cuanto a la variable estudiada.

6. Se elaboraron baremos independientes de acuerdo al sexo.

\section{REFERENCIAS BIBLIOGRÁFICAS}

1. American Psychiatric Association (2000). Diagnostic and statistical manual of mental disorders (DSM-IV-TR). 4.a ed.Washington, DC.

2. Arias, A., Ayuso, L., Gil, G. y González, I. (2009). Alteración del Comportamiento en la Escuela $(A C E)$. Madrid: TEA Ediciones. 
3. Campo-Arias, A. y Oviedo, H. (2008). Propiedades psicométricas de una escala: la consistencia interna. En Revista de Salud Pública, 10, 5, 831-839. Recuperado en: http://redalyc.uaemex.mx/src/inicio/ArtPdfRed.jsp?iCve $=42210515$, el día 03.12.10, 10:48 p.m.

4. Elosua, P. (2003). Sobre la validez de los tests. En Psicothema, 15, 2, 315321. Recuperado en: http://redalyc.uaemex.mx/redalyc/src/inicio/ArtPdfRed. jsp?iCve $=72715225$, el día 03.12.10, 10:48 p.m.

5. Ezpeleta, L. (2001). La entrevista diagnóstica en niños y adolescentes. Madrid: Síntesis.

6. Ferrando, P. y Anguiano, C. (2010). El análisis factorial como técnica de investigación en psicología. En Papeles del psicólogo, 31, 1, 18-33. Recuperado en: http://redalyc. uaemex.mx/src/inicio/ArtPdfRed.jsp?iCve=77812441003, el día 07.12.10, 23:24 p.m.

7. García, L. (2008). Psicología del desarrollo. Lima: Fondo Editorial de la Universidad Nacional Mayor de San Marcos.

8. Hernández, R., Fernández, C. y Baptista, P. (2003). Metodología de la investigación 3. ${ }^{\text {a }}$ ed. México D.F.: McGraw Hill.

9. León, I. y Montero, O. (2005). Clasificación y descripción de las metodologías de investigación en psicología. En Journal of Clinical and Health Psychology. 2, 3, 503-508. Recuperado en: http://redalyc.uaemex.mx/redalyc/pdf/337/33720308.pdf, el día 04.12.10, 21:33 p.m.

10. Marsh, E. y Graham, S. (2002). Clasificación y tratamiento de la psicopatología infantil. En Caballo, V. y Simon, M. (Ed.), Manual de psicología clínica infantil y del adolescente: trastornos generales (29-56). Madrid, España: Pirámide.

11. Organización Mundial de la Salud (1992). Décima clasificación de los trastornos mentales y del comportamiento: descripciones clínicas y pautas para el diagnóstico. Madrid: Meditor.

12. Prieto, G. y Delgado, A. (2010). Fiabilidad y Validez. En Papeles del psicólogo, 31, 1, 67-74. Recuperado en: http://redalyc.uaemex.mx/src/inicio/ArtPdfRed. jsp?iCve $=77812441007$, el día 02.12.10, 22:17 p.m. 